\title{
Why stripes? Spontaneous formation of inhomogeneous structures due to elastic interactions
}

\author{
D.I. Khomskii ${ }^{1}$ and K.I. Kugel ${ }^{2}$ \\ 1 Laboratory of Solid State Physics, Groningen University, Nijenborgh 4, 9747 AG Groningen, The Netherlands \\ ${ }^{2}$ Institute for Theoretical and Applied Electrodynamics, Russian Academy of Sciences, Izhorskaya Str. 13/19, 127412 \\ Moscow, Russia
}

We argue that elastic interactions between ions in different valence states can play an essential role in stabilization of stripes (or $2 D$ "sheets") in doped oxides. These interactions are in general long-range and anisotropic (attractive in certain directions and repulsive in others). This can naturally give rise to stripe-like structures in insulating materials. We illustrate this general idea with certain specific examples and show that the situation can be described by the Ising model with anisotropic interactions. The case of anisotropic impurities, relevant e.g. for manganites, is also briefly discussed. 1

PACS numbers: 71.28.+d, 61.50.Ah, 64.75.+g

The notion of stripes became recently one of the most important concepts in the physics of doped Mott insulators. There are many theoretical [1] 3] and experimental [5 8] indications that they indeed arise in doped transition metal oxides; there exist also serious arguments that they may play an important role in high-temperature superconductivity [1, 9, 10].

Despite very intense studies in this field, the origin of stripes in real materials is far from being well understood. Following the pioneering work 1 the main attention is paid to the purely electronic and magnetic mechanisms of stripe formation [2]3]. However the existence of stripes in this approach is still controversial 任.

When discussing the origin of stripes in doped oxides, one usually starts with Mott insulators with an integer number of electrons per site forming local magnetic moments. Generically their ground state is antiferromagnetic. In this case, one argues that with doping a superstructure may develop consisting of antiferromagnetic domains, the doped charge carriers being localised at the domain walls. This reflects the general tendency: instability of a homogeneous state in doped strongly correlated electron systems toward phase separation 111 14. Stripe phases are possible manifestations of this tendency.

Why are stripe phases better than any other forms of phase separation? This is the main question, which, in our opinion, still did not find a satisfactory answer. One

\footnotetext{
${ }^{1}$ Accepted for publication in Europhysics Letters
}

can argue that in systems with only short-range interaction, such as the conventional Hubbard or $t-J$ models, one would rather expect a total phase separation into two phases: an antiferromagnetic insulator without any holes $(n=1)$ and another phase - metallic and probably ferromagnetic - containing all the holes; this state allows to gain the full kinetic energy of holes, and simultaneously it costs a minimum of surface energy.

To stabilize the stripe phase, one often invokes longrange Coulomb interaction which prevents the large-scale phase separation [9, 10]. However, for the strong longrange repulsion we would rather expect not stripes but a sort of Wigner crystal, in which the doped carriers keep as far away from each other as possible. In this case, stripes, and even more so two-dimensional sheets observed e.g. in manganites [5, 6], are definitely less favourable. One then argues that stripes would be stable in the intermediate case. And although this scenario is of course not excluded, it seems that some important physical factors are missing from such a picture. 2

We argue that there is indeed one such factor, which exists in all real systems and which can, in principle, stabilize the stripe and sheet phases. This factor is the long-range elastic interaction. We consider below the insulating materials (nickelates, overdoped manganites) with the so called filled stripes (one hole per site); the situation in cuprates with presumably half-filled stripes may be more complicated, although we believe that the factors invoked below can also play an important role there.

When we dope a stoichiometric system, e.g. substitute $\mathrm{Ca}$ for $\mathrm{La}$ in $\mathrm{LaMnO}_{3}$, we create $\mathrm{Mn}^{4+}$ ions in the $\mathrm{Mn}^{3+}$ matrix. Or, in the overdoped $\mathrm{La}_{1-x} \mathrm{Ca}_{x} \mathrm{MnO}_{3}$ with $x>$ 0.5 (this is the situation, in which the stripe, or rather sheet phases were observed in manganites [5, [0]), one can speak of a certain amount of $\mathrm{Mn}^{3+}$ ions in the $\mathrm{Mn}^{4+}$ matrix.

Classically, $\mathrm{Mn}^{3+}$ ions differ from $\mathrm{Mn}^{4+}$ (or $\mathrm{Ni}^{2+}$ from $\mathrm{Ni}^{3+}$ in nickelates $\left.[7,8]\right)$ not only by their charge, but also by their ionic radius. This factor is usually ignored in the conventional treatment of stripes. In contrast, we

\footnotetext{
${ }^{2}$ Note also that in nickelates and manganites, in contrast to cuprates, the metal-centered stripes would not correspond to the antiferromagnetic domain walls inherent to the conventional theories [1] 4 .
} 
pay the main attention here just to this factor (which, of course, should be considered alongside with the other factors, usually accounted for $11,4,9,10 \|$ ).

As is known from the theory of elasticity, when we create a lattice distortion (in simple case - a dilatation, e.g. by cutting out a small sphere of radius $A$ and substituting it by the sphere of radius $A^{\prime} \neq A$ ), we also create a field of lattice strains, which is long-range and, in general, anisotropic [15,16]. This leads to a long-range interaction between impurities $\left(\sim 1 / R^{3}\right)$, which depends on the elastic constants of the medium and on the shape of impurities.

The most important point is that typically this interaction resembles a quadrupole-quadrupole interaction, being repulsive in certain directions but attractive in others. This long-range attraction opens a possibility to form regular structures - clusters of impurities forming one-dimensional objects (needles or stripes) or twodimensional structures (lamellae or sheets); this can be one of the mechanisms stabilizing stripe or sheet phases.

The interaction of dilatation impurities in crystals vanishes in isotropic media, but it is nonzero in crystals. Thus, in crystals with cubic anisotropy it is given by 15]

$$
V\left(\vec{r}, \vec{r}^{\prime}\right)=-C d Q_{1} Q_{2} \frac{\Gamma(\vec{n})}{|\vec{r}-\vec{r}|^{3}}
$$

where $C$ is a constant of the order of unity, $\vec{r}-\vec{r}^{\prime}=$ $\left|\vec{r}-\vec{r}^{\prime}\right| \cdot \vec{n}, Q_{1}$ and $Q_{2}$ are the "strengths" of impurities $\left(Q_{i} \sim\left(v_{i}-v_{0}\right)\right.$ where $v_{i}$ is the volume of the impurity and $v_{0}$ is the corresponding volume of the matrix), and

$$
d=c_{11}-c_{12}-2 c_{44},
$$

where $c_{i j}$ are the elastic moduli of the crystal. The angular dependence of the interaction (1) is determined by a function of the direction cosines of the vector $\vec{R}=\vec{r}_{1}-\vec{r}_{2}$ :

$$
\Gamma(\vec{n})=n_{x}^{4}+n_{y}^{4}+n_{z}^{4}-\frac{3}{5} .
$$

From (11)-(3) we can see that the interaction between impurities is long-range $\left(\sim 1 / R^{3}\right)$ and has different signs in different directions. Thus, for the situation with $d>0$ it is attractive along [100], [010] and [001] directions $\left(\Gamma([100])=\frac{2}{5}\right)$ and is repulsive along [110], [011] etc. $\left(\Gamma([110])=-\frac{1}{10}\right)$ and along the cube diagonals [111]: $\Gamma([111])=-\frac{4}{15}$, and vice versa for $d<0$. Thus, if we put a few dilatation impurities in a cubic crystal, they will attract each other along certain directions (e.g. [100], [010], [010]) and repel along others, thus causing a formation of inhomogeneous structures (stripes, sheets etc.)

\footnotetext{
${ }^{3}$ Note also that in contrast to the Coulomb forces, these elastic interactions are not screened (except by the "mirror forces" due to the surface of the crystal 115]).
}

In real systems (not the weakly anisotropic crystals considered above as an example), the ratios of the interaction constants between different neighbors may differ from those given above; thus, e.g., in bcc iron the interaction with [011] neighbor is stronger than with [001] one 16]. But again, as in the example treated above, the interactions have different signs in different directions. Thus, we can consider the situation of a cubic lattice with the nearest-neighbor $(n n)$ and next-nearestneighbor ( $n n n)$ interaction of opposite signs as a typical one, treating the ratio of these couplings as an arbitrary parameter. This naturally leads to the lattice gas, or the Ising model of the type

$$
H=-J \sum_{\langle i j\rangle=n n} \sigma_{i} \sigma_{j}+J^{\prime} \sum_{\langle i j\rangle=n n n} \sigma_{i} \sigma_{j}+\text { const }
$$

where $\sigma_{i}= \pm 1$; the density at site $i$ is $n_{i}=\frac{1}{2}\left(1+\sigma_{i}\right)$, and the model has to be considered for fixed density $n=\left\langle n_{i}\right\rangle$ (or fixed "magnetisation"). We treat below the case of a cubic lattice.

For $J, J^{\prime}>0$ (which corresponds to $d>0$ in Eq. (1)) we have the following solutions:

1. Totally phase separated state, in which all the "impurities" (or electrons) form one big cluster. The energy of this state (per impurity) in a simple cubic lattice is $E_{\text {ph.sep. }}=-J z_{n n} / 2+J^{\prime} z_{n n n} / 2=-3 J+6 J^{\prime}$. Here $z$ are the corresponding numbers of nearest and next nearest neighbours.

2. Occupied sites forming $2 D$ sheets parallel to $(x y)$, $(x z)$ or $(y z)$ planes, such that the distance between them exceeds one lattice spacing. The energy of such state is $E_{\text {sheet }}=-2 J+2 J^{\prime}$.

3. The $1 D$ stripes in $x, y$ or $z$ directions, which do not cross or approach one another to one lattice spacing. The energy of such a state is $E_{\text {stripe }}=-J$.

One can easily show that all the other possible structures have higher energy.

By comparing the energies of these states, we see that the totally phase separated state will be stable if $J^{\prime} / J<\frac{1}{4}$; for $\frac{1}{4}<J^{\prime} / J<\frac{1}{2}$ the $2 \mathrm{D}$ "sheets" are stable, and for $J^{\prime} / J>\frac{1}{2}$ the $1 \mathrm{D}$ stripes would give a minimum of the energy. Thus, we see that the sheet or stripe phases appear quite naturally if the dominant interaction is the elastic interaction between impurities which is intrinsically anisotropic and long-range. For $d<0$, we similarly obtain, instead of the vertical, the diagonal stripes, but in this case, we should also include the interaction with third neighbors.

For low electron (or impurity) density in 3D case the lamellae phase is formed by parallel sheets; the distance between them in model (4) with only $n n$ and $n n n$ interactions is arbitrary, provided only that it exceeds the lattice spacing. Longer-range interactions will make this arrangement regular, but for weak interaction of this kind the discommensurations could be easily formed.

The situation is a bit more complicated for the stripe phase. In layered materials, it will be similar to that 
with the sheets in $3 D$ crystals (parallel equally spaced stripes). In $3 D$ crystals there may in principle exist mutually perpendicular stripes. Again, one may expect that longer-range interaction can make these stripes parallel; this question, however, goes beyond the scope of our treatment. Thus, we see that the elastic interaction of the "spherical" impurities in crystals may quite naturally lead to the formation of inhomogeneous structures- $1 D$ stripes, $2 D$ sheets or $3 D$ phase-separated clusters, which can be described by the Ising-like model (14) [17]. Note also that this process does not require the atomic diffusion: it is realized by the electron hopping between, say, $M n^{3+}$ and $M n^{4+}$ (with the corresponding lattice relaxation).

The problem of formation of regular structures, when we have two components with different atomic volumes, is well-known in the physics of segregating alloys. Theoretical studies (see e.g. 16]) have shown that the shape of inclusions of one phase in another is to a large extent determined by the elastic strains: the shape is such as to minimize the total elastic energy. As is shown in the corresponding studies, the best way is to create the second phase in the form of infinitely thin layers having certain specific orientation in given matrix. In the corresponding alloys it gives rise to the so called Guinier-Preston zones 18,19]: inclusions of the second phase appear as a regular array of parallel platelets. One can speculate that the physics of the stabilization of stripe phases (actually sheets) in $\mathrm{La}_{1-x} \mathrm{Ca}_{x} \mathrm{MnO}_{3}$ at $x=0.67,0.75$, etc. [5.6] is the same, and that it is just these long-range anisotropic elastic forces which stabilize such structures. This is supported by the fact that this stripe charge ordering is obtained in the insulating materials at temperatures above the eventual magnetic ordering; thus, the often invoked magnetic mechanisms play here a minor role, if any. The inclusion of the electron hopping will, of course, tend to destabilize these ordered structures, which could survive if the hopping integrals are not too large.

The situation may be different (and actually much richer) if the "impurities" are not simple dilatation centers ("spheres"), but are anisotropic ("ellipsoids"). This is the typical situation with Jahn-Teller ions, e.g. $\mathrm{Mn}^{3+}$ "impurities" in overdoped manganites $\mathrm{R}_{1-x} \mathrm{Ca}_{x} \mathrm{MnO}_{3}(\mathrm{R}$ $=\mathrm{La}, \mathrm{Pr}, \mathrm{Nd})$ for $x>0.5$. The interaction between such impurities also decays as $1 / R^{3}$ and has different signs depending on both the relative position of such centres in a crystal and on the orientation of corresponding orbitals (i.e. local distortions) 20,21]. Thus, for example, for two quadrupolar ions along the $z$-axis with the electron density elongated parallel to $x, y$, or $z$ axes, as is the case of $e_{g}$-ions like $\mathrm{Mn}^{3+}$ one obtains from the general expressions 20] the interaction in the form

$$
\begin{aligned}
V= & \frac{\left(c_{11}+c_{44}\right)}{8 \pi\left(c_{11}+2 c_{44}\right) R^{3}}\left\{5 \sigma_{z z}^{(1)} \sigma_{z z}^{(2)}+\right. \\
& \left.+2\left(\sigma_{x x}^{(1)} \sigma_{x x}^{(2)}+\sigma_{y y}^{(1)} \sigma_{y y}^{(2)}\right)\right\}+
\end{aligned}
$$

$$
+\frac{1}{4 \pi R^{3}}\left(2 \sigma_{z z}^{(1)} \sigma_{z z}^{(2)}-\sigma_{x x}^{(1)} \sigma_{x x}^{(2)}-\sigma_{y y}^{(1)} \sigma_{y y}^{(2)}\right)
$$

where $c_{11}$ and $c_{44}$ are elastic moduli, and $\sigma_{\alpha \alpha}$ is a stress tensor such that e.g. for the center with the occupied orbital $3 z^{2}-r^{2}$ we have $\sigma_{z z}=1, \sigma_{x x}=\sigma_{y y}=-1 / 2$ (and corresponding expressions for $z \rightarrow x, y)$.

From this expression one can see that e.g. two $3 z^{2}-r^{2}$ orbitals along $z$-axis strongly repel one another, whereas $3 z^{2}-r^{2}$ and $3 x^{2}-r^{2}$ (or $3 y^{2}-r^{2}$ ) along the same direction attract. This can quite naturally explain the well-known orbital structure of undoped $\mathrm{LaMnO}_{3}$ (cf. [22]), and also a special stability of the $\mathrm{Mn}^{3+}$-planes, with the corresponding orbital ordering, in lightly doped manganites, e.g. $\mathrm{La}_{1-x} \mathrm{Sr}_{x} \mathrm{MnO}_{3}, x \sim 1 / 8$ 25 and in $\mathrm{Pr}_{1-x} \mathrm{Ca}_{x} \mathrm{MnO}_{3}, x \sim 1 / 4$ [26]. With the assumption of a checkerboard charge ordering it also gives the orbital ordering of $\mathrm{CE}$ type observed in $\mathrm{R}_{1-x} \mathrm{Ca}_{x} \mathrm{MnO}_{3}$ at $x=0.5$ 23,24. Indeed, in both cases the orbitals of the neighbors along $x$ and $y$ directions are mutually orthogonal (e.g. $3 x^{2}-r^{2}$ and $3 y^{2}-r^{2}$ orbitals); according to (5) they have an attractive interaction which stabilizes these structures. One can also show that the interaction along diagonals ([110] direction) is attractive for the parallel orbitals $3 x^{2}-r^{2}$ or $3 y^{2}-r^{2}$ but repulsive for the perpendicular ones $\left(3 x^{2}-r^{2}\right.$ at one site and $3 y^{2}-r^{2}$ at the other); this gives extra stability to the observed orbital ordering in undoped $\mathrm{LaMnO}_{3}$, and these diagonal interactions nearly cancel for the CE-type phase in the $x=0.5$ system. The same factors may favor single stripes ("Wigner crystal") [6] as compared to the bi-stripes [5].

Summarizing, we suggest that the elastic interactions, always present in systems with mixed valence, e.g. in doped Mott insulators, may play an important role in stabilizing particular types of inhomogeneous structures, such as stripes or two-dimensional sheets. These interactions decay rather slowly $\left(\sim R^{-3}\right)$ and are anisotropic, being repulsive in some directions but attractive in others. Thus, the interaction of dilatation impurities in weakly anisotropic cubic crystals has different signs along [100], [010], and [001] directions and along face or body diagonals. Such an interaction may quite naturally lead to the formation of stripes or sheets in manganites or nickelates. Similar interactions in the case of anisotropic (Jahn-Teller) ions can produce analogous inhomogeneous structures with specific orbital ordering. The importance of lattice effect in the stripe formation is supported by the observation of a large isotope effect on the stripe ordering temperature [27,28]. Thus, we can conclude that the elastic or, more generally, electron-lattice interactions may be very important in providing a mechanism for stripe formation in doped systems.

We are grateful to S.-W. Cheong, M. V. Eremin, A. Ya. Fishman, J. Hill, S. Kivelson, F. Kusmartsev, V. Ya. Mitrofanov, and G. Sawatzky for very useful discussions.

The work was supported by INTAS (grants 97-0963 and 97-11954), the Russian Foundation for Basic Re- 
search (grant 00-15-96570), the Netherlands Foundation for the Fundamental Research of Matter (FOM), and the Netherlands Organization for the Advancement of Pure Research (NWO).

[1] J. Zaanen and O. Gunnarsson, Phys. Rev. B 40, 7391 (1989).

[2] R. White and D. J. Scalapino, Phys. Rev. Lett. 80, 1272 (1998).

[3] B. P. Stoiković, Z. G. Yu, A. R. Bishop, A. H. Castro Neto, and N. Grønbech-Jensen, Phys. Rev. Lett. 82, 4679 (1999).

[4] C. S. Hellberg and E. Manousakis, Phys. Rev. Lett. 83,132, (1999).

[5] S. Mori, C. H. Chen, and S.-W. Cheong, Nature (London) 392, 473 (1998).

[6] P. G. Radaelli, D. E. Cox, L. Capogna, S.-W. Cheong, and M. Marezio, Phys. Rev. B 59, 14440 (1999).

[7] C. H. Chen, S.-W. Cheong, and A. S. Cooper, Phys. Rev. Lett. 71, 2461 (1993).

[8] J. M. Tranquada, D. J. Buttrey, V. Sachan, and J. E. Lorenzo, Phys. Rev. Lett. 73, 1003 (1994).

[9] V. J. Emery and S. A. Kivelson, Physica C 209, 597 (1993); V. J. Emery, S. A. Kivelson, and O. Zachar, Phys. Rev. B 56, 6120 (1997).

[10] G. Seibold, C. Castellani, C. Di Castro, and M. Grilli, Phys. Rev. B 58, 13506 (1998).

[11] P. B. Visscher, Phys. Rev. B 10, 943 (1974).

[12] É. L. Nagaev, Usp. Fiz. Nauk 166, 833 (1996) [PhysicsUspekhi 39, 781 (1996)].

[13] A. Moreo, S. Yunoki, and E. Dagotto, Science 283, 2034 (1999).

[14] D. I. Khomskii, Physica B 280, 325 (2000).

[15] Eshelby, J. D. The continuum theory of lattice defects. in Solid State Physics, eds. F. Seitz and D. Turnbull, 3, 79 (Academic Press, New York, 1956).

[16] A. G. Khachaturyan, Theory of phase transformations and the structure of solid solutions (Nauka, Moscow, 1974).

[17] The conclusion that stripes can appear in an Ising model with longer-range interaction was reached by U. Löw, V. J. Emery, K. Fabricius, and S. A. Kivelson, Phys. Rev. Lett., 72, 1918 (1994), who, however, had in mind different physics - that of frustrated phase separation due to long-range Coulomb forces [9]. The physics closest to ours was invoked by F. V. Kusmartsev, Phys. Rev. Lett. $\mathbf{8 4}, 530$ (2000), who suggested that the effective latticemediated attraction between polarons plus the Coulomb repulsion may lead to a formation of polaron strings of finite length. He, however, did not consider the anisotropic nature of the effective elastic interactions, which is crucial for our treatment.

[18] G. D. Preston, Proc. Roy. Soc. (London) A 167, 526 (1938).

[19] A. Guinier, Annales de Physique 12, 161-237 (1939).
[20] M. V. Eremin, A. Yu. Zavidonov, and B. I. Kochelaev, Zh. Éksp. Teor. Fiz. 90, 537 (1986) [ Sov. Phys.-JETP 63, 312 (1986)].

[21] M. A. Ivanov, V. Ya. Mitrofanov, and A. Ya. Fishman, Fiz. Tv. Tela (Sov.Phys.-Solid State) 20, 3023 (1978).

[22] P. Novák, J. Phys. Chem. Solids 302357 (1969); 31125 (1970).

[23] E. O. Wollan, and W. C. Koehler, Phys. Rev. 100, 545 (1955).

[24] Z. Jirák, S. Krupička, Z. Šimša, M. Dlouhá, and S. Vratislav, J. Magn. Magn. Mater. 53, 153 (1985).

[25] T. Mizokawa, D. Khomskii, and G. A. Sawatzky, Phys. Rev. B 61, R3776 (2000).

[26] M. v. Zimmermann, C. S. Nelson, J. P. Hill, D. Gibbs, M. Blume, D. Casa, B. Keimer, Y. Murakami, C.-C. Kao, C. Venkataraman, T. Gog, Y. Tomioka, and Y. Tokura, cond-mat/0007231 (2000).

[27] A. Lanzara, Guo-meng Zhao, N. L. Saini, A. Bianconi, K. Conder, H. Keller, and K. A. Müller, J. Phys.: Condens. Matter 11, L541 (1999).

[28] D. Rubio Temprano, J. Mesot, S. Janssen, K. Conder, A. Furrer, H. Mutka, K. A. Müller, Phys. Rev. Lett. 84, 1990 (2000). 